\title{
Die Praxis des U-Haft Vollzugs - Daten und Fakten
}

Heribert Ostendorf

Tab. 1: Bestand und Art des Vollzuges*

- Bundesrepublik Deutschland gesamt -

(2008)

\begin{tabular}{|l|r|r|r|}
\hline Art des Vollzugs & insgesamt & männlich & weiblich \\
\hline insgesamt & 72259 & 68418 & 3841 \\
\hline $\begin{array}{l}\text { Untersuchungshaft } \\
\text { Jugendstrafe }\end{array}$ & 11577 & 10949 & 628 \\
$\begin{array}{l}\text { Freiheitsstrafe } \\
\text { (einschließlich Ersatz- } \\
\text { freiheitsstrafe) }\end{array}$ & 52333 & 49474 & 2859 \\
$\begin{array}{l}\text { Sicherungsverwah- } \\
\text { rung }\end{array}$ & 461 & 458 & 3 \\
$\begin{array}{l}\text { sonstige Freiheitsent- } \\
\text { ziehung } \\
\text { davon: -Strafarrest } \\
\text { - Abschiebungshaft }\end{array}$ & 1826 & 1705 & 121 \\
\hline
\end{tabular}

Quelle: Statistisches Bundesamt, Rechtspflege, Bestand der Gefangenen und Verwahrten, Stichtag 30.11.2008

* ohne die vorübergehend Abwesenden ( $n=7559$ ) laut monatlicher Belegungsstatistik des BMJ.

Die Untersuchungshaftpopulation ist zu 94,6\% männlich und $\mathrm{zu}$ $5,4 \%$ weiblich. Diese Geschlechterverteilung entspricht der im Strafvollzug.

Tab. 2: Belegungsentwicklung in der BRD

\begin{tabular}{|l|c|c|c|c|c|c|}
\hline Jahr & $\begin{array}{c}\text { Gefange- } \\
\text { ne inse- } \\
\text { samt* }\end{array}$ & $\begin{array}{c}\text { U-Gefan- } \\
\text { gene }\end{array}$ & $\begin{array}{c}\text { Anteil } \\
\text { U-Haft } \\
\text { in } \%\end{array}$ & $\begin{array}{c}\text { Erwach- } \\
\text { sene }\end{array}$ & $\begin{array}{c}\text { Heran- } \\
\text { wach- } \\
\text { sende }\end{array}$ & $\begin{array}{c}\text { Jugendli- } \\
\text { che }\end{array}$ \\
\hline 1980 & 51889 & 14929 & 28,8 & 12267 & 2040 & 622 \\
\hline 1981 & 53597 & 15636 & 29,2 & 12743 & 2141 & 752 \\
\hline 1982 & 57277 & 16539 & 28,9 & 13638 & 2040 & 861 \\
\hline 1983 & 55816 & 14600 & 26,2 & 12269 & 1714 & 617 \\
\hline 1984 & 53166 & 13303 & 25,0 & 11313 & 1513 & 477 \\
\hline 1985 & 50255 & 12254 & 24,4 & 10346 & 1446 & 462 \\
\hline 1986 & 45666 & 11373 & 24,9 & 9687 & 1319 & 367 \\
\hline 1987 & 44903 & 11527 & 26,1 & 9922 & 1188 & 417 \\
\hline 1988 & 44804 & 11.703 & 26,1 & 10314 & 1010 & 379 \\
\hline 1989 & 43900 & 12222 & 27,8 & 10811 & 1087 & 324 \\
\hline 1990 & 44355 & 14070 & 31,7 & 12380 & 1309 & 381 \\
\hline $1991 * *$ & 45892 & 14415 & 31,4 & 12480 & 1532 & 403 \\
\hline 1992 & 49106 & 18370 & 37,4 & 15354 & 2323 & 693 \\
\hline 1993 & 59833 & 21787 & 36,4 & 18516 & 2359 & 912 \\
\hline $1994 * * *$ & 60289 & 20203 & 33,5 & 17159 & 2210 & 834 \\
\hline 1995 & 61108 & 19787 & 32,3 & 16696 & 2199 & 892 \\
\hline 1996 & 64680 & 20440 & 31,6 & 17274 & 2232 & 934 \\
\hline 1997 & 68029 & 19935 & 29,3 & 16848 & 2154 & 933 \\
\hline 1998 & 69917 & 19049 & 27,2 & 15979 & 2216 & 854 \\
\hline 1999 & 69214 & 17661 & 25,5 & 14633 & 2135 & 893 \\
\hline 2000 & 70252 & 17524 & 24,9 & 14501 & 2120 & 903 \\
\hline & & & & & & \\
\hline
\end{tabular}

\begin{tabular}{|l|c|c|c|c|c|r|}
\hline Jahr & $\begin{array}{c}\text { Gefange- } \\
\text { ne insge- } \\
\text { samt* }\end{array}$ & $\begin{array}{c}\text { U-Gefan- } \\
\text { gene }\end{array}$ & $\begin{array}{c}\text { Anteil } \\
\text { U-Haft } \\
\text { in \% }\end{array}$ & $\begin{array}{c}\text { Erwach- } \\
\text { sene }\end{array}$ & $\begin{array}{c}\text { Heran- } \\
\text { wach- } \\
\text { sende }\end{array}$ & $\begin{array}{c}\text { Jugendli- } \\
\text { che }\end{array}$ \\
\hline 2001 & 70203 & 17431 & 24,8 & 14411 & 2097 & 923 \\
\hline 2002 & 70977 & 16853 & 23,7 & 14175 & 1864 & 814 \\
\hline 2003 & 79153 & 16785 & 21,2 & 14206 & 1837 & 742 \\
\hline 2004 & 79452 & 15783 & 19,8 & 13512 & 1586 & 685 \\
\hline 2005 & 78664 & 15228 & 19,3 & 13029 & 1547 & 652 \\
\hline 2006 & 76629 & 13330 & 17,3 & 11414 & 1319 & 597 \\
\hline 2007 & 72656 & 12357 & 17,0 & 10608 & 1205 & 544 \\
\hline 2008 & 72259 & 11577 & 16,0 & 9915 & 1166 & 496 \\
\hline
\end{tabular}

Quelle: Strafvollzugsstatistiken der Jahre 1980 bis 2002, Statistisches Bundesamt Fachserie 10/Reihe 4.2, ab 2003 Statistisches Bundesamt, Rechtspflege, Bestand der Gefangenen und Verwahrten Stichtag bis 2002 jeweils 31.12, ab 2003 jeweils 30.11.

* Straf- und Untersuchungshäftlinge

** rüheres Bundesgebiet ohne Hamburg

*** Gesamtdeutschland

Die Zahl der U-Gefangenen ist in den letzten Jahren deutlich zurückgegangen, gegenüber 1994 um 42,7\%, bei Erwachsenen um 42,2\%, bei Heranwachsenden um 47,2\% und bei Jugendlichen um 40,5\%. Auch der prozentuale Anteil an den Gefangenen hat mit 16,0\% im Jahr 2008 den niedrigsten Wert. Die frühere Kritik „Es wird viel zu schnell in der BRD verhaftet" 1 sowie die einschränkende Rechtsprechung der Beschwerdeinstanzen und die Haftprüfungen durch die Oberlandesgerichte haben offensichtlich eine rechtsstaatlich heilsame Wirkung gezeigt.

Tab. 3: Jugendliche, Heranwachsende und Erwachsene in Untersuchungshaft

Jugendliche, Heranwachsende und Erwachsene in Untersuchungshaft pro 100000 der Altersgruppe

\begin{tabular}{|c|c|c|c|c|c|c|}
\hline \multirow{2}{*}{ Jahr } & \multicolumn{2}{|c|}{ Jugendliche } & \multicolumn{2}{c|}{ Heranwachsende } & \multicolumn{2}{c|}{ Erwachsene } \\
\cline { 2 - 7 } & absolut & $\begin{array}{c}\text { pro } \\
100000\end{array}$ & absolut & $\begin{array}{c}\text { pro } \\
100000\end{array}$ & absolut & $\begin{array}{c}\text { Pro } \\
100000\end{array}$ \\
\hline 1970 & 761 & 23,4 & 1754 & 71,2 & 10523 & 24,8 \\
\hline 1980 & 822 & 19,3 & 2124 & 61,7 & 12267 & 27,6 \\
\hline 1990 & 381 & 15,1 & 1309 & 53,2 & 12380 & 25,0 \\
\hline 1995 & 892 & 24,9 & 2199 & 85,4 & 16696 & 26,4 \\
\hline 2000 & 903 & 24,7 & 2120 & 74,3 & 14501 & 22,7 \\
\hline 2005 & 652 & 17,2 & 1545 & 53,9 & 13029 & 20,0 \\
\hline 2006 & 597 & 16,4 & 1319 & 45,0 & 11414 & 17,5 \\
\hline 2007 & 544 & 15,5 & 1205 & 41,0 & 10608 & 16,2 \\
\hline
\end{tabular}

Quelle: Statistisches Jahrbuch 2008, Tabellen 2.8, 10.17 Gebiet: bis 1990 alte Länder; ab 2000 Gesamtdeutschland

Die Heranwachsenden weisen die höchste U-Haftrate auf, fast 3-mal so viel wie bei den Jugendlichen und Erwachsenen. Auch bei Berücksichtigung demographischer Veränderungen sind die U-Haftraten für alle 3 Altersgruppen stark gesunken. 
Tab. 4: Haftgründe (2007)

\section{Haftgründe*}

- alte Bundesländer und Berlin -

Personen mit Untersuchungshaft insgesamt: 26793

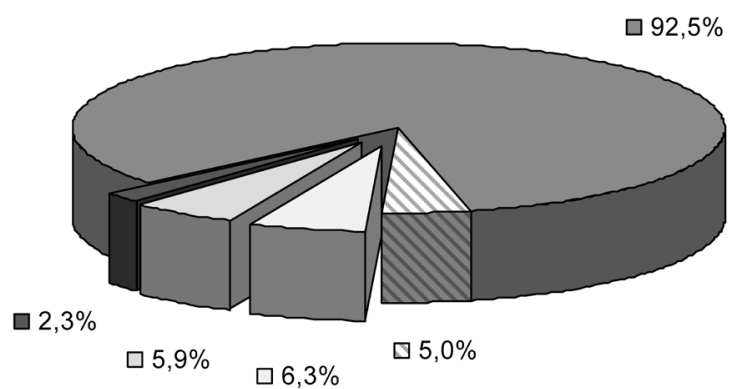

$\square$ Flucht/Fluchtgefahr 24 776, darunter $\square$ Hauptverhandlungshaft 1345
$\square$ Verdunklungsgefahr $1675 \quad \square$ Wiederholungsgefahr 1589
$\square$ Schwere der Tat 608

Quelle: Strafverfolgungsstatistik 2007 (Fachserie 10 Reihe 3), hrsg. vom Statistischen Bundesamt Wiesbaden, Tab. 6.1;

Strafgerichte 2007 (Fachserie 10 Reihe 2.3), hrsg. vom Statistischen Bundesamt Wiesbaden, Tab. 2.1

* auch mehrere nebeneinander möglich; deshalb ergibt das Gesamt der Haftgründe mehr als 100\%.

Bei den Haftgründen dominiert der Haftgrund der Flucht/Fluchtgefahr, obwohl faktisch eine Flucht auf Grund der heute möglichen und internationalen Fahndungsmethoden immer schwieriger geworden ist.

Als Flucht/Fluchtgefahr wird auch die sog. Hauptverhandlungshaft registriert. Die Anzahl der Verfahren mit sog. Hauptverhandlungshaft gem. $\$ 127 \mathrm{~b}$ StPO ist stark abnehmend und in den Bundesländern sehr unterschiedlich ${ }^{2}$ :

Tab. 5: Hauptverhandlungshaft gem. $\ 127 \mathrm{~b}$ StPO

\begin{tabular}{|c|c|}
\hline Jahr & $\begin{array}{c}\text { Anzahl Beschuldigter in } \\
\text { Hauptverhandlungshaft } \\
(\mathbb{1 2 7 b} \text { StPO })\end{array}$ \\
\hline 2005 & 2294 \\
\hline 2006 & 1821 \\
\hline 2007 & 1345 \\
\hline
\end{tabular}

Quelle: Strafgerichte (Fachserie 10 Reihe 2.3), Tab. 2.1; hrsg. vom Statistischen Bundesamt Wiesbaden

Ebenfalls nicht gesondert ausgewiesen werden Verhaftungen gem. \$230 Abs. 2 StPO, die dementsprechend auch unter dem Haftgrund „Flucht/Fluchtgefahr“ registriert werden. ${ }^{3}$

Bemerkenswert ist, dass Haftbefehle gem. $\$ 112$ Abs. 3 StPO (Schwere der Tat) in den letzten Jahren deutlich angestiegen sind: Im Jahr 2000275 Haftbefehle (0,7\%), im Jahr 20061124 Haftbefehle $(4,6 \%)$. Zu den offiziellen Haftgründen, die im Haftbefehl angegeben werden, kommen sog. apokryphe Haftgründe, verdeckte-unechte Haftgründe. ${ }^{4}$ Dies gilt insbesondere im Jugendstrafrecht, wo das Erziehungsstrafrecht seit eh und je eine scheinbare Legitimation lieferte, was heute als Krisenintervention bezeichnet wird, womit contra legem der Haftgrund der Wiederholungsgefahr des $\mathbb{} 112 \mathrm{a}$ StPO ausgedehnt wird. ${ }^{5}$ Weitere Gründe sind ein mit einkalkulierter „Geständniszwang " ${ }^{6}$ sowie das fürsorgliche Anliegen, mit Rücksicht auf die Erfahrung des Freiheitsentzuges im Strafurteil noch eine Bewährungsstrafe zu ermöglichen.

Tab. 6: Belegungsentwicklung von Straf- und Untersuchungshaft in den alten und neuen Bundesländern im Vergleich

\begin{tabular}{|c|c|c|c|c|}
\hline Jahr & $\begin{array}{c}\text { Alte Bundes- } \\
\text { länder }\end{array}$ & $\begin{array}{c}\text { Alte Bundes- } \\
\text { länder }\end{array}$ & $\begin{array}{c}\text { Neue Bun- } \\
\text { desländer }\end{array}$ & $\begin{array}{c}\text { Neue Bun- } \\
\text { desländer }\end{array}$ \\
\hline & Strafhaft & U-Haft & Strafhaft & U-Haft \\
\hline $1992 *$ & 30506 & 16413 & 1721 & 1957 \\
\hline 1993 & 34585 & 18897 & 3461 & 2890 \\
\hline 1994 & 35509 & 17056 & 4577 & 3147 \\
\hline 1995 & 35737 & 16725 & 5584 & 3062 \\
\hline 1996 & 37833 & 17424 & 6407 & 3016 \\
\hline 1997 & 40624 & 16954 & 7470 & 2981 \\
\hline 1998 & 42440 & 16246 & 8428 & 2803 \\
\hline 1999 & 42910 & 14921 & 8643 & 2740 \\
\hline 2000 & 43103 & 14729 & 9625 & 2795 \\
\hline 2001 & 43237 & 14897 & 9535 & 2534 \\
\hline 2002 & 44316 & 14615 & 9808 & 2238 \\
\hline 2003 & 51601 & 14623 & 10767 & 2162 \\
\hline 2004 & 52722 & 13651 & 10947 & 2132 \\
\hline 2005 & 52489 & 13444 & 10867 & 1844 \\
\hline 2006 & 52744 & 11792 & 10555 & 1538 \\
\hline 2007 & 50566 & 11019 & 9733 & 1338 \\
\hline 2008 & 51212 & 10236 & 9470 & 1341 \\
\hline & & & & \\
\hline
\end{tabular}

Quelle: Strafvollzugsstatistiken der Jahre 1992 bis 2002, Statistisches Bundesamt Fachserie 10/Reihe 4.2, ab 2003 Statistisches Bundesamt, Rechtspflege, Bestand der Gefangenen und Verwahrten Stichtag bis 2002 jeweils 31.12, ab 2003 jeweils 30.11

* ohne Hamburg und Brandenburg

Der gemeinsame „Höhepunkt“ in der Belegung der U-Haft waren in den alten wie neuen Bundesländern die Jahre 1994-1996. Seitdem ist die Belegung deutlich zurückgegangen.

Tab. 7: Straf- und Untersuchungsgefangene im europäischen Vergleich $(2007 / 2008)$

\begin{tabular}{|c|c|c|c|c|c|}
\hline & Stichtag & $\begin{array}{l}\text { Gefangenen- } \\
\text { rate (pro } \\
100.000 \text { der } \\
\text { Wohnbev. }\end{array}$ & $\begin{array}{c}\text { Anteil } \\
\text { von U- } \\
\text { Gefange- } \\
\text { nen (\%) }\end{array}$ & $\begin{array}{c}\text { Inhaftierungsrate } \\
\text { (Inhaftierungen pro } \\
100.000 \text { der } \\
\text { Wohnbev. (2005) }\end{array}$ & $\begin{array}{l}\text { Indikator für die } \\
\text { durch- } \\
\text { schnittliche In- } \\
\text { haftierungszeit } \\
\text { (Monate) (2005) }\end{array}$ \\
\hline Albanien & 1.6 .2008 & 159 & 39,0 & - & - \\
\hline Belgien & 17.6 .2008 & 93 & 36,1 & 145,7 & 7,3 \\
\hline $\begin{array}{l}\text { Bosnien u. } \\
\text { Herzegowina }\end{array}$ & 30.4 .2008 & 67 & 19,4 & - & - \\
\hline Bulgarien & 1.1 .2008 & 134 & 9,3 & - & - \\
\hline Dänemark & 4.9.2008 & 63 & 34,4 & 340,3 & 2,6 \\
\hline Deutschland & 31.3 .2008 & 91 & 17,0 & 149,1 & $6,8(2001)$ \\
\hline EnglandWales & 26.9 .2008 & 153 & 16,5 & - & - \\
\hline Estland & 1.1.2008 & 259 & 26,4 & $=$ & - \\
\hline Finnland & 1.1 .2008 & 64 & 14,0 & 143,5 & 6,2 \\
\hline Frankreich & 1.9.2007 & 91 & 27,7 & 135,4 & 8,4 \\
\hline Georgien & 31.1 .2008 & 415 & 16,1 & - & - \\
\hline Griechenland & 1.11.2007 & 99 & 28,6 & - & - \\
\hline Irland & 26.10 .2007 & 76 & 20,0 & 258,8 & 3,5 \\
\hline
\end{tabular}




\begin{tabular}{|c|c|c|c|c|c|}
\hline & Stichtag & $\begin{array}{l}\text { Gefangenen- } \\
\text { rate (pro } \\
100.000 \text { der } \\
\text { Wohnbev. }\end{array}$ & \begin{tabular}{|c|} 
Anteil \\
von U- \\
Gefange- \\
nen (\%)
\end{tabular} & $\begin{array}{c}\text { Inhaftierungsrate } \\
\text { (Inhaftierungen pro } \\
100.000 \text { der } \\
\text { Wohnbev. (2005) }\end{array}$ & $\begin{array}{l}\text { Indikator für die } \\
\text { durch- } \\
\text { schnittliche In- } \\
\text { haftierungszeit } \\
\text { (Monate) (2005) }\end{array}$ \\
\hline Island & 1.9 .2008 & 44 & 7,1 & 108,2 & 4,3 \\
\hline Italien & 1.1 .2008 & 83 & 58,1 & 152,9 & - \\
\hline Kroatien & 1.7 .2007 & 93 & 29,9 & 292,8 & 2,9 \\
\hline Lettland & 1.1 .2008 & 288 & 26,6 & - & - \\
\hline Litauen & 1.1 .2008 & 234 & 12,1 & 335,6 & 8,4 \\
\hline Luxemburg & 1.9 .2007 & 155 & 42,0 & 281,6 & 6,3 \\
\hline Malta & 1.7 .2007 & 95 & 31,3 & 154,9 & - \\
\hline Mazedonien & 1.3 .2008 & 107 & 15,9 & 358,2 & 4,0 \\
\hline Moldawien & 1.9 .2006 & 246 & 20,6 & 631,2 & - \\
\hline Montenegro & 1.9 .2003 & 108 & 38,1 & - & - \\
\hline Niederlande & 31.8 .2008 & 100 & 34,7 & 273,0 & 4,2 \\
\hline Nordirland & 29.9 .2008 & 88 & 37,3 & 279,9 & 2,2 \\
\hline Norwegen & 1.8 .2008 & 69 & 21,1 & 253,2 & 3,1 \\
\hline Österreich & 1.8 .2008 & 95 & 20,0 & 172,7 & 7,8 \\
\hline Polen & 31.8 .2008 & 222 & 11,3 & 247,9 & 9,9 \\
\hline Portugal & 15.9 .2008 & 103 & 28,9 & 53,1 & $9,9(2001)$ \\
\hline Rumănien & 16.9 .2008 & 126 & 10,0 & 73,2 & 29,1 \\
\hline Russland & 1.9 .2008 & 630 & 15,6 & 478,4 & - \\
\hline Schottland & 26.9 .2008 & 155 & 21,2 & 753,9 & 2,1 \\
\hline Schweden & 1.10.2007 & 74 & 22,2 & 240,7 & 3,6 \\
\hline Schweiz & 5.9 .2007 & 76 & 37,9 & - & - \\
\hline Serbien & 1.3 .2007 & 116 & 30,4 & 180,6 & - \\
\hline Slowakei & 1.1 .2008 & 148 & 23,2 & 131,8 & - \\
\hline Slowenien & 1.9 .2007 & 66 & 25,1 & 258,5 & 6,2 \\
\hline Spanien & 26.9.2008 & 157 & 25,0 & 89,3 & 18,6 \\
\hline Tschechien & 1.1 .2008 & 182 & 11,9 & 185,9 & - \\
\hline Turkei & 1.4 .2008 & 135 & 60,9 & 161,4 & 6,7 \\
\hline Ukraine & 1.1 .2008 & 323 & 21,5 & - & - \\
\hline Ungarn & 2.9 .2008 & 149 & 28,9 & 124,2 & 16,9 \\
\hline Weißrussland & 1.1.2006 & 426 & 19,6 & - & $=$ \\
\hline Zypern & 31.8 .2008 & 83 & 15,4 & 261,7 & $2,9(2001)$ \\
\hline
\end{tabular}

Quellen: Gefangenenraten: International Center for Prison Studies, World Prison Brief, InternetPublikation, http//www.kcl.ac.uk/depsta/rel/icps/worldbrief/world_brief.html (Abfrage 3.11.2008); Inhaftierungsraten: Council of Europe, Hrsg., Annual Penal Statistics - SPACE I- 2006 , Strasbourg 2007, S. 55,58

\section{Entnommen Greifswalder Inventar für Strafvollzug, Lehrstuhl für Krimi-} nologie

Ein Ländervergleich ist schwierig. Einmal müssen die Gefangenenraten vor dem Hintergrund unterschiedlicher Strafgesetze und vor allem unterschiedlicher Kriminalitätsbelastungen betrachtet werden, zum anderen findet z. T. ein „Austausch“ zwischen U-Haftraten und Straf-Haftraten statt. Unberücksichtigt bleiben auch Unterbringungen von Straftätern in psychiatrischen Einrichtungen. Nichtsdestotrotz sind die hohen Gefangenenraten in den Baltischen Staaten sowie in Osteuropa mehr als auffallend. Umgekehrt fallen die Skandinavischen Staaten sowie die Schweiz, Irland und insbesondere Island durch ihre niedrigen Gefangenenraten auf. Im Hinblick hierauf sind Bemühungen um eine weitere Senkung der Gefangenenraten und hier insbesondere der U-Haftraten in Deutschland angezeigt.
Tab. 8: Untersuchungshaftraten in den Ländern nach Jugendstrafrecht und nach allgemeinem Strafrecht (2004)

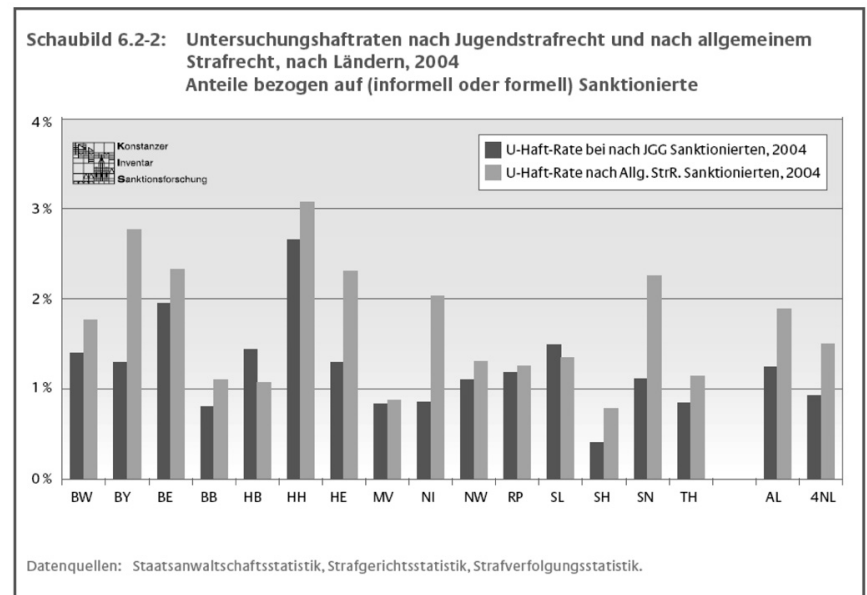

Quelle: Zweiter Periodischer Sicherheitsbericht, S. 552

Auch der innerstaatliche Ländervergleich ist vor dem Hintergrund unterschiedlicher Kriminalitätsbelastungen schwierig. So ist nicht verwunderlich, dass die Staatstaaten Berlin und Hamburg aufgrund der hohen Kriminalitätsdichte erhöhte U-Haftzahlen aufweisen. Aber bereits die im Vergleich hierzu geringe U-Haftrate von Bremen ist insoweit bemerkenswert. Von den Flächenländern fallen Bayern, Hessen, Niedersachsen sowie Sachsen mit hohen U-Haftraten bei Erwachsenen auf, umgekehrt Schleswig-Holstein mit einer besonders niedrigen U-Haftrate. Weiterhin auffällig ist die höhere U-Haftrate im Jugendstrafrecht im Vergleich zum Erwachsenenstrafrecht im Saarland. Ganz offensichtlich ist hier ein unterschiedliches justizielles Entscheidungsverhalten ausschlaggebend, eine ,justizielle U-Haftpolitik“.

Im Hinblick auf den Verhältnismäßigkeitsgrundsatz - U-Haft „darf nicht angeordnet werden, wenn sie zu der Bedeutung der Sache und der zu erwartenden Strafe oder Maßregel der Besserung und Sicherung außer Verhältnis steht" ( $\mathbb{} 112$ Abs. 1 S. 2 StPO) - ist der Prozentsatz von 52,6 der Fälle, in denen eine Freiheitsstrafe ohne Bewährung angeordnet wurde, problematisch. Auf der einen Seite darf im Strafurteil nicht auf Freiheitsstrafe erkannt werden, nur weil bereits U-Haft vollstreckt wurde - entsprechend dem sarkastischen Kalauer „U-Haft schafft Rechtskraft“. Auf der anderen Seite wäre in vielen Fällen im Hinblick auf die zu erwartende Strafe offensichtlich U-Haft vermeidbar gewesen. Dies gilt nach einer Untersuchung bei jugendlichen/heran-wachsenden U-Gefangenen. Im Jahr 1989 haben bei diesen nur 39,6 \%, im Jahr 1990 nur 33,9 \% und im Jahr 1991 nur 36,2 \% eine Jugend- oder Freiheitsstrafe ohne Bewährung erhalten. ${ }^{7}$ Besonders auffällig ist der Ausgang von Verkehrsstrafverfahren mit U-Haft. Bemerkenswert ist weiterhin, dass sich bei Sexualdelikten der angenommene dringende Tatverdacht zu 5,5\% nicht bestätigt hat.

Die höchsten U-Haftraten weisen Verfahren wegen Tötungsdelikten auf (s. auch $\ 112$ Abs. 3 StPO). Bemerkenswert ist der hohe Anteil beim qualifizierten Diebstahl ( $\mathbb{S} \mathbb{S} 243,244$ StGB), der noch höher liegt als bei den Sexualdelikten. Die Dauer korreliert mit der Schwere des Tatvorwurfs: Bei Verfahren wegen Tötungsdelikten dauerte die U-Haft im Jahr 2007 zu 36,3 \%, im Jahr 2006 zu 43,2 \% über 12 Monate; bei Verfahren wegen eines Diebstahls dauerte die U-Haft im Jahr 2007 zu 2,1 \%, im Jahr 2006 zu 2,5 \% über 12 Monate. 
Tab. 9: Abgeurteilte mit Untersuchungshaft nach der Art der Entscheidung - alte Bundesländer und Berlin - (2007)

\begin{tabular}{|c|c|c|c|c|c|c|c|c|c|}
\hline \multirow{3}{*}{ Delikte } & Abgeur- & \multicolumn{8}{|c|}{ ART DER SANKTION } \\
\hline & insgesamt & \multicolumn{2}{|c|}{$\begin{array}{c}\text { Freiheitsstrafe* ohne } \\
\text { Bewährung }\end{array}$} & \multicolumn{2}{|c|}{$\begin{array}{l}\text { Freiheitsstrafe }{ }^{*} \text { mit } \\
\text { Bewährung }\end{array}$} & \multicolumn{2}{|c|}{$\begin{array}{c}\text { Strafarrest, } \\
\text { Geldstrafe, } \\
\text { Zuchtmittel, } \\
\text { Maßregel, } \\
\text { Erziehungsmaßregel }\end{array}$} & \multicolumn{2}{|c|}{$\begin{array}{c}\text { Freispruch, } \\
\text { Einstellung, } \\
\text { Absehen v. Strafe }\end{array}$} \\
\hline & abs. & abs. & $\%$ & abs. & $\%$ & abs. & $\%$ & abs. & $\%$ \\
\hline Straftaten & 26715 & 14056 & 52,6 & 8861 & 33,2 & 3051 & 11,4 & 747 & 2,8 \\
\hline $174-184 b$ & 1213 & 795 & 65,5 & 313 & 25,8 & 39 & 3,2 & 66 & 5,5 \\
\hline $211-222$ & 559 & 491 & 87,8 & 11 & 2,0 & 36 & 6,4 & 21 & 3,8 \\
\hline $223-231$ & 2535 & 1221 & 48,2 & 914 & 36,1 & 326 & 12,8 & 74 & 2,9 \\
\hline $242-248 c$ & 6844 & 3676 & 53,7 & 2212 & 32,3 & 787 & 11,5 & 169 & 2,5 \\
\hline 243,244 & 2871 & 1658 & 57,8 & 973 & 33,9 & 167 & 5,8 & 73 & 2,5 \\
\hline $\begin{array}{c}249-255 \\
316 a\end{array}$ & 2749 & 1859 & 67,6 & 724 & 26,4 & 69 & 2,5 & 97 & 3,5 \\
\hline $263-266 b$ & 2082 & 1005 & 48,3 & 779 & 37,4 & 222 & 10,7 & 76 & 3,6 \\
\hline Verkehr & 352 & 104 & 29,5 & 127 & 36,1 & 108 & 30,7 & 13 & 3,7 \\
\hline BtMG & 5884 & 3543 & 60,2 & 1932 & 32,8 & 333 & 5,7 & 76 & 1,3 \\
\hline andere & 1647 & & & & & & & & \\
\hline
\end{tabular}

Quelle: Strafverfolgungsstatistik (Fachserie 10/Reihe 3) 2007, Tab. 6.2 (eigene Berechnungen)

* Freiheitsstrafe i.w.S. (einschließlich Jugendstrafe, Strafarrest)

Anm.: Entscheidungen nach §§ 59, 27, 45 JGG wurden nicht berücksichtigt.

Tab. 10: Abgeurteilte nach der Deliktsstruktur und der Dauer der Untersuchungshaft - alte Bundesländer und Berlin - (2007)

\begin{tabular}{|c|c|c|c|c|c|c|c|c|}
\hline \multirow{2}{*}{\begin{tabular}{|l|} 
Delikte \\
Straftaten insgesamt
\end{tabular}} & \multicolumn{2}{|l|}{ Abgeurteilte } & \multirow{2}{*}{$\begin{array}{c}\text { mit U-Haft } \\
\% \\
\text { (bez. auf } \\
\text { Sp.1) }\end{array}$} & \multicolumn{5}{|c|}{$\begin{array}{l}\text { Dauer der Untersuchungshaft } \\
\text { (in Monaten) }\end{array}$} \\
\hline & absolut & absolut & & $<1$ & $\begin{array}{c}>1 \\
\text { bis } \\
3\end{array}$ & $\begin{array}{c}>3 \\
\text { bis } \\
6\end{array}$ & $\begin{array}{l}>6 \\
\text { bis } \\
12\end{array}$ & über 12 \\
\hline Spalte & 1 & 2 & 3 & 4 & 5 & 6 & 7 & 8 \\
\hline Straftaten insgesamt & 1111577 & 26715 & 2,4 & 7569 & 6340 & 6650 & 4672 & 1562 \\
\hline $174-184 b$ & 10910 & 1213 & 11,1 & 208 & 196 & 360 & 322 & 130 \\
\hline $211-222$ & 1468 & 559 & 38,1 & 16 & 20 & 73 & 247 & 203 \\
\hline $223-231$ & 130250 & 2535 & 1,9 & 860 & 577 & 549 & 414 & 145 \\
\hline $242-248 c$ & 195988 & 6844 & 3,5 & 1997 & 2232 & 1688 & 800 & 149 \\
\hline 243,244 & 22323 & 2871 & 12,9 & 613 & 924 & 868 & 404 & 74 \\
\hline $249-256,316 a$ & 14465 & 2749 & 19,0 & 447 & 649 & 876 & 613 & 186 \\
\hline $263-266 \mathrm{~b}$ & 219733 & 2082 & 0,9 & 708 & 527 & 443 & 304 & 103 \\
\hline Verkehr & 239326 & 352 & 0,1 & 214 & 64 & 43 & 25 & 6 \\
\hline BtMG & 64237 & 5884 & 9,2 & 1271 & 956 & 1767 & 1442 & 461 \\
\hline andere & 70851 & 1647 & 2,3 & & & & & \\
\hline
\end{tabular}

Quelle: Statistisches Bundesamt Strafverfolgungsstatistik 2007 (Fachserie 10/Reihe 3), Tab. 2.1, 6.1 und 6.2 (eigene Berechnungen) 
Tab. 11: Dauer der Untersuchungshaft (2007)

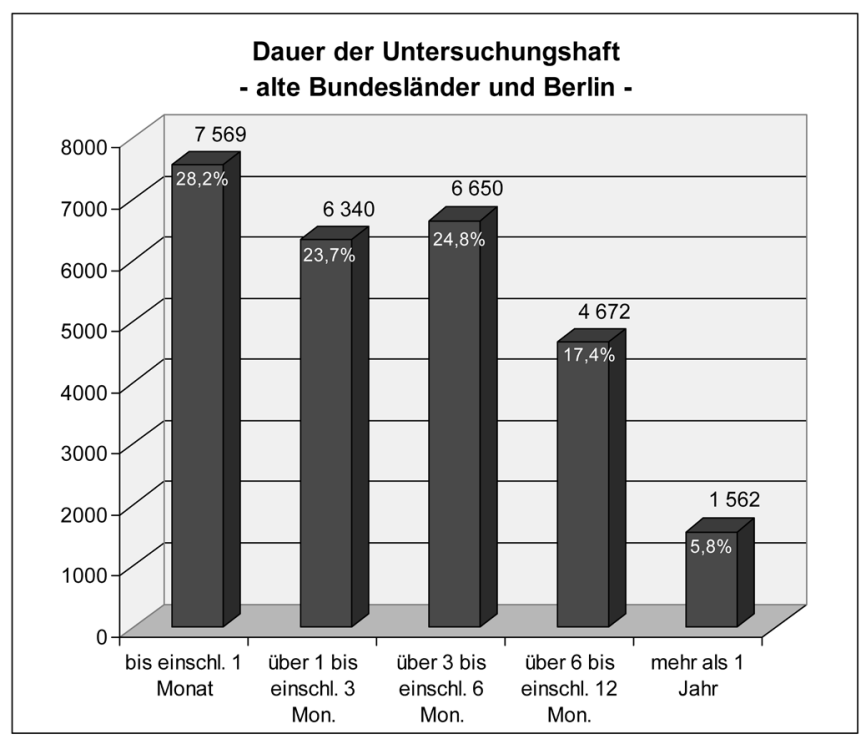

Quelle: Strafverfolgungsstatistik 2007, hrsg. vom Statistischen Bundesamt Wiesbaden.

Die Dauer der U-Haft erscheint zu lang. Damit wird nicht dem Beschleunigungsgebot entsprochen, das mit Art. 2 Abs. 2 S. 2 GG, dem Grundrecht auf Freiheit, verfassungsrechtlich abgesichert ist. ${ }^{8}$ Mit $\ 121$ Abs. 1 StPO macht der Gesetzgeber deutlich, dass eine U-Haft nur ausnahmsweise länger als 6 Monate dauern darf. Diese Ausnahmeregelung wird mit 23,2 \% der Verfahren, die über 6 Monate dauern und hiervon $5,8 \%$, die über 12 Monate dauern, nicht eingehalten.

Tab. 12: Dauer der Untersuchungshaft in \% der abgeurteilten Untersuchungsgefangenen in den Jahren 1980, 1990, 2000, 2006 und 2007

\begin{tabular}{|l|r|r|r|r|r|}
\hline Haftdauer & 1980 & 1990 & 2000 & 2006 & 2007 \\
\hline bis 1 Monat & 40,7 & 37,8 & 35,6 & 25,8 & 28,2 \\
\hline 1 bis 3 Monate & 26,6 & 24,0 & 23,3 & 24,1 & 23,7 \\
\hline 3 bis 6 Monate & 18,6 & 19,5 & 22,4 & 25,6 & 24,8 \\
\hline 6 bis 12 Monate & 11,2 & 13,0 & 14,5 & 18,4 & 17,4 \\
\hline mehr als 12 Monate & 3,4 & 4,9 & 4,3 & 6,1 & 5,8 \\
\hline $\begin{array}{l}\text { U-Haft insgesamt } \\
\text { (N=100\%) }\end{array}$ & 37238 & 27553 & 36683 & 24352 & 26793 \\
\hline $\begin{array}{l}\text { davon mehr als 6 } \\
\text { Monate }\end{array}$ & 14,6 & 17,9 & 18,8 & 24,5 & 23,2 \\
\hline
\end{tabular}

Quelle: Statistisches Bundesamt Strafverfolgungsstatistiken der jew. Jahrgänge (Fachserie 10/Reihe 3)

Die Dauer der U-Haft hat sich seit 1980 deutlich verlängert. Im Jahr 1980 betrug die U-Haftdauer zu 40,7 \% nur bis zu 1 Monat, im Jahr 2007 verringerte sich dieser Prozentsatz auf 28,2 \%. Umgekehrt war die Haftzeit im Jahr 1980 in 3,4 \% der Verfahren mehr als 12 Monate lang, im Jahr 2007 aber in 5,8 \% der Verfahren. Allerdings wird dieses Ergebnis durch die verringerte Anzahl der U-Haft relativiert, da davon auszugehen ist, dass vormals eine kurze U-Haft in Verfahren registriert wurde, in denen heute überhaupt keine U-Haft angeordnet wird. Diese Relativierung gilt aber nicht für die längeren
U-Haftzeiten, für die nur die Erhöhung des Schwierigkeitsgrades der Ermittlungen angeführt werden kann.

Tab. 13: Belegung, Belegungsfähigkeit und das Verhältnis von Straf- und Untersuchungshaft im Bundesländervergleich (2008)

\begin{tabular}{|l|r|r|r|r|}
\hline Land & $\begin{array}{r}\text { Belegungs- } \\
\text { fähigkeit }\end{array}$ & $\begin{array}{r}\text { Belegung } \\
\text { insgesamt }\end{array}$ & $\begin{array}{r}\text { Straf- } \\
\text { haft }\end{array}$ & $\begin{array}{r}\text { Unter- } \\
\text { suchungs- } \\
\text { haft }\end{array}$ \\
\hline Baden-Wü. & 8418 & $7504(89 \%)$ & 6063 & 1441 \\
\hline Bayern & 11827 & $12047(102 \%)$ & 9599 & 2448 \\
\hline Berlin & 5140 & $4934(96 \%)$ & 4255 & 679 \\
\hline Brandenburg & 2326 & $1758(76 \%)$ & 1542 & 216 \\
\hline Bremen & 748 & $610(82 \%)$ & 532 & 78 \\
\hline Hamburg & 3169 & $1905(60 \%)$ & 1537 & 368 \\
\hline Hessen & 5848 & $5041(86 \%)$ & 4142 & 899 \\
\hline Mecklenburg-V. & 1587 & $1398(88 \%)$ & 1163 & 235 \\
\hline Niedersachsen & 7423 & $6326(85 \%)$ & 5452 & 874 \\
\hline Nordrhein-Westf. & 18323 & $17379(95 \%)$ & 14681 & 2698 \\
\hline Reinl.-Pfalz & 3608 & $3469(96 \%)$ & 3070 & 399 \\
\hline Saarland & 886 & $784(88 \%)$ & 654 & 130 \\
\hline Sachsen & 4079 & $3563(87 \%)$ & 3119 & 444 \\
\hline Sachsen-Anh. & 2467 & $2165(88 \%)$ & 1931 & 234 \\
\hline Schleswig-Holst. & 1734 & $1449(84 \%)$ & 1227 & 222 \\
\hline Thüringen & 2130 & $1927(90 \%)$ & 1715 & 212 \\
\hline Alte Länder* & 67124 & $61448(92 \%)$ & 51212 & 10236 \\
\hline Neue Länder & 12589 & $10811(86 \%)$ & 9470 & 1341 \\
\hline
\end{tabular}

Quelle: Statistisches Bundesamt, Rechtspflege, Bestand der Gefangenen und Verwahrten 2008 (Stichtag 30.11.2008), Spalte 4 eigene Berechungen

* früheres Bundesgebiet einschl. Berlin-Ost

Formal rechnerisch ist nur - noch - der Freiheitsentzug in Bayern überbelegt. Im Hinblick darauf, dass nicht immer alle Gefangenen „am Platze“ sind, z.B. wegen Verschub, wegen Urlaub bei Strafgefangenen, ist der Prozentsatz für eine Überbelegung niedriger anzusetzen, zumindest ab $95 \% .{ }^{9} \mathrm{Da}$ hinsichtlich der Belegungsfähigkeit Straf- und U-Haft zusammengefasst sind, geben die Zahlen hinsichtlich der tatsächlichen Situation im U-Haftvollzug keinen Aufschluss. Eine Länderumfrage zum Stichtag 31.3.2009 hatte für folgende Bundesländer dieses Ergebnis:

Tab. 13a: Belegungsfähigkeit und tatsächliche Belegung in der UHaft nach Bundesländern (Stichtag 31.3.2009)

\begin{tabular}{|l|c|c|}
\hline Bundesland & $\begin{array}{l}\text { Belegungsfähigkeit } \\
\text { in der U-Haft }\end{array}$ & $\begin{array}{l}\text { Tatsächliche Bele- } \\
\text { gung in der U-Haft }\end{array}$ \\
\hline Brandenburg & 419 & 197 \\
\hline Bremen & 126 & 71 \\
\hline Hamburg & 596 & 338 \\
\hline Hessen & 1322 & 901 \\
\hline Sachsen-Anhalt & 367 & 226 \\
\hline Schleswig-Holstein & 408 & 223 \\
\hline
\end{tabular}

Die anderen Bundesländer sahen sich aufgrund einer Mischkalkulation aus Straf- und U-Haft hinsichtlich der Belegungsfähigkeit nicht in der Lage, hierzu Angaben zu machen. Dementsprechend wurde die Frage „Stellen die U-Haft-Vollzugseinreichtungen 
a) eigenständige Anstalten dar,

b) sind sie Teilanstalten bzw. Abteilungen in einer Strafvollzugsanstalt oder

c) wird der U-Haft-Vollzug in einem Haus mit Strafgefangenen durchgeführt? “ wie folgt beantwortet:

Tab. 13b: Art der U-Haftvollzugseinrichtungen in den einzelnen Bundesländern (Stichtag 31.3.2009)

\begin{tabular}{|l|c|c|c|}
\hline Bundesland & $\begin{array}{c}\text { Antwort a) } \\
\text {-eigenständige } \\
\text { Anstalten- }\end{array}$ & $\begin{array}{c}\text { Antwort b) } \\
\text {-Teilanstal- } \\
\text { ten/Abtei- } \\
\text { lungen- }\end{array}$ & $\begin{array}{c}\text { Antwort c) } \\
\text {-in einem } \\
\text { Haus mit } \\
\text { Strafgefan- } \\
\text { genen- }\end{array}$ \\
\hline Baden-Württemberg & ja & ja & ja \\
\hline Bayern & nein & ja & ja \\
\hline Berlin & nein & ja & ja \\
\hline Brandenburg & nein & ja & ja \\
\hline Bremen & nein & ja & ja \\
\hline Hamburg & ja & ja & ja \\
\hline Hessen & nein & ja & ja \\
\hline $\begin{array}{l}\text { Mecklenburg-Vor- } \\
\text { pommern }\end{array}$ & nein & ja & ja \\
\hline Niedersachsen & ja & ja & nein \\
\hline Nordrhein-Westfalen & nein & ja & ja \\
\hline Rheinland-Pfalz & nein & ja & ja \\
\hline Saarland & nein & ja & ja \\
\hline Sachsen & nein & ja & ja \\
\hline Sachsen-Anhalt & nein & ja & nein \\
\hline Schleswig-Holstein & ja & ja & ja \\
\hline Thüringen & nein & ja & ja \\
\hline
\end{tabular}

Insgesamt wird U-Haft in der Bundesrepublik in 145 Anstalten vollzogen.

Tab. 14: Ausländeranteil in der Untersuchungshaft

\begin{tabular}{|r|r|r|}
\hline Jahr & absolut & in \% \\
\hline 1998 & 10154 & 51,2 \\
\hline 1999 & 9019 & 48 \\
\hline 2000 & 8623 & 48 \\
\hline 2001 & 6423 & 37,3 \\
\hline 2002 & 8077 & 46,2 \\
\hline 2003 & 7563 & 44,2 \\
\hline 2004 & 7169 & 45,4 \\
\hline 2005 & 6954 & 45,2 \\
\hline $2006 *$ & 6483 & 45,3 \\
\hline $2007^{*}$ & 5569 & 43,2 \\
\hline $2008^{*}$ & 5297 & 42,9 \\
\hline
\end{tabular}

Quelle: Bundesministerium der Justiz, Stichtag jeweils 31.03.

* Datenerhebung aus 15 Bundesländern ohne Schleswig-Holstein

Der Ausländeranteil ist zwar in den letzten Jahren leicht, im Vergleich zu 1998 deutlich gesunken, ist aber nominal deutlich im Vergleich zu dem registrierten (!) Ausländeranteil in der Bundesrepublik deutlich überhöht (1.10.2008: 8,8 \%). Auch gegenüber dem prozen- tualen Anteil der Nichtdeutschen an der polizeilich registrierten Kriminalität - im Jahr 2007 21,4 \% - ist der Anteil der U-Gefangenen überproportional hoch. Hierfür können schwergewichtige Straftaten, insbesondere aber die „leichtere“ Annahme von Haftgründen ursächlich sein. ${ }^{10}$

Prof. Dr. Heribert Ostendorf, Forschungsstelle für Jugendstrafrecht und Kriminalprävention an der Christian-Albrechts-Universität Kiel

\section{Fußnoten:}

1 Forum des Deutschen Anwaltsvereins 1983, s. Stab NJW 1983, 1039; s. auch Bleckmann StV 1995, 552; Deckers NJW 1994, 2261.

2 S. auch Gensen die Hauptverhandlungshaft (\$127b II StPO), 2005, S. 157, 158.

$3 \mathrm{Zu}$ einer empirischen Erhebung siehe Triltsch, Die Zwangsmittel des $\$ 230$ Abs. 2 StPO unter besonderer Berücksichtigung des Haftbefehls, 2009. Hiernach wurden im Durchschnitt in Schleswig-Holstein im Zeitraum 2002-2007 10 Haftbefehle gem. $\$ 230$ Abs. 2 StPO ausgestellt (S. 133), wobei die Haftdauer zwar überwiegend kurz (bis 30 Tage), bei $32 \%$ aber über 30 Tage ausfiel (S. 191).

4 Schlothauer/Weider, Untersuchungshaft, 3. Aufl., Rn $633 \mathrm{ff}$.

5 Heinz BewH 1987, 5, 24; Ostendorf, JGG, 8. Aufl., $\$ 72$ Rn 4.

6 Seebode, Der Vollzug der Untersuchungshaft, 1985, S. 66.

7 Jehle, Entwicklung der Untersuchungshaft bei Jugendlichen und Heranwachsenden vor und nach der Wiedervereinigung, hrsg. vom BMJ, 1995, S. 79.

8 So nachdrücklich BVerfG StV 2006, 249 m.w.N.

9 In der Literatur wird bereits ab $90 \%$ von Überbelegung ausgegangen, s. Walter, Strafvollzug, 2. Aufl., Rn 106 m.w.N.

10 Dies trifft nach einer empirischen Untersuchung gerade für illegal sich aufhaltende junge Ausländer zu, bei denen $61 \%$ wegen ausländertypischer Bagatelldelikte in U-Haft genommen wurden, s. Staudinger, Ilka, Untersuchungshaft bei jungen Ausländern, 2001, S. 121; s. weiterhin Henniger Nichtdeutsche Beschuldigte im Jugendstrafverfahren, 2003, S. 206.

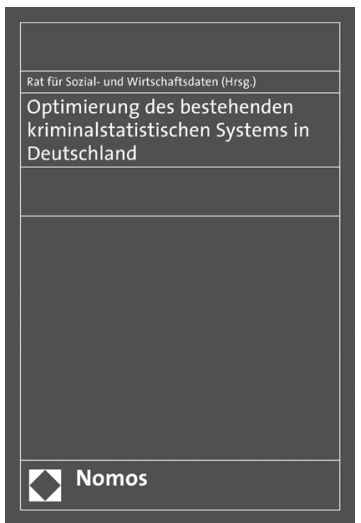

\section{Optimierung des bestehenden kriminalstatistischen Systems in Deutschland} Empfehlungen der Arbeitsgruppe „Optimierung des bestehenden kriminalstatistischen Systems" unter dem Vorsitz von Prof. Dr. Wolfgang Heinz, Universität Konstanz Herausgegeben vom Rat für Sozial- und Wirtschaftsdaten 2009, 156 S., brosch., 29,- €, ISBN 978-3-8329-4793-4 Erscheint ca. Dezember 2009

\section{Nomos}

Bitte bestellen Sie im Buchhandel oder versandkostenfrei unter $\rightarrow$ www.nomos-shop.de 